Bull. Austral. Math. Soc.

VoL. 76 (2007) [1-13]

\title{
ON INEQUALITIES OF HILBERT'S TYPE
}

\section{YongJin Li ANd Bing He}

By introducing the function $1 /(\min \{x, y\})$, we establish several new inequalities similar to Hilbert's type inequality. Moreover, some further unification of Hardy-Hilbert's and Hardy-Hilbert's type integral inequality and its equivalent form with the best constant factor are proved, which contain the classic Hilbert's inequality as special case.

\section{INTRODUCTION}

If $f, g$ are real functions such that $0<\int_{0}^{\infty} f^{2}(x) d x<\infty$ and $0<\int_{0}^{\infty} g^{2}(x) d x<\infty$, then we have (see Hardy, Littlewood and Polya [4])

$$
\int_{0}^{\infty} \int_{0}^{\infty} \frac{f(x) g(y)}{x+y} d x d y<\pi\left\{\int_{0}^{\infty} f^{2}(x) d x \int_{0}^{\infty} g^{2}(x) d x\right\}^{1 / 2}
$$

where the constant factor $\pi$ is the best possible. Inequality (1.1) is the well known Hilbert's inequality. Inequality (1.1) had been generalised by Hardy-Riesz (see [3]) in 1925 as:

$$
\text { If } f, g \geqslant 0, p>1,(1 / p)+(1 / q)=1,0<\int_{0}^{\infty} f^{p}(x) d x<\infty \text { and } 0<\int_{0}^{\infty} g^{q}(x) d x
$$
$<\infty$, then

$$
\begin{aligned}
& \int_{0}^{\infty} \int_{0}^{\infty} \frac{f(x) g(y)}{x+y} d x d y<\frac{\pi}{\sin (\pi / p)}\left\{\int_{0}^{\infty} f^{p}(x) d x\right\}^{1 / p}\left\{\int_{0}^{\infty} g^{q}(x) d x\right\}^{1 / q} \\
& \int_{0}^{\infty}\left(\int_{0}^{\infty} \frac{f(x)}{x+y} d x\right)^{p} d y<\left[\frac{\pi}{\sin (\pi / p)}\right]^{p} \int_{0}^{\infty} f^{p}(x) d x
\end{aligned}
$$

where the constant factor $\pi /(\sin (\pi / p))$ is the best possible. When $p=q=2,(1.2)$ reduces to (1.1), Inequality (1.2) is Hardy-Hilbert's integral inequality, which is important in analysis and its applications(see [7]). It has been studied and generalised in many directions by a number of mathematicians (see $[1,2,6,8,10])$.

Recently, by introducing some parameters, Yang (see [11]) obtained the following inequalities:

\section{Received 5th June, 2006}

The work was partially supported by the Emphases Natural Science Foundation of Guangdong Institution of Higher Learning, College and University (No. 05Z026). The authors would like to thank the anonymous referee for his or her suggestions and corrections.

Copyright Clearance Centre, Inc. Serial-fee code: 0004-9727/07 \$A2.00+0.00. 
THEOREM 1.1. If $p>1,1 / p+1 / q=1, f, g \geqslant 0, f \in L^{p}(0, \infty), g \in L^{q}(0, \infty)$ and $\|f\|_{p},\|g\|_{q}>0$, then for $0<\lambda<\min \{1 / p, 1 / q\}$, one has the following two equivalent inequalities:

$$
\begin{gathered}
\int_{0}^{\infty} \int_{0}^{\infty} \frac{|x-y|^{\lambda-1}}{(\min \{x, y\})^{\lambda}} f(x) g(y) d x d y<\left[B\left(\lambda, \frac{1}{q}-\lambda\right)+B\left(\lambda, \frac{1}{p}-\lambda\right)\right]\|f\|_{p}\|g\|_{q} \\
\left\{\int_{0}^{\infty}\left(\int_{0}^{\infty} \frac{|x-y|^{\lambda-1}}{(\min \{x, y\})^{\lambda}} f(x) d x\right)^{p} d y\right\}^{1 / p} \\
<\left[B\left(\lambda, \frac{1}{q}-\lambda\right)+B\left(\lambda, \frac{1}{p}-\lambda\right)\right]\|f\|_{p}
\end{gathered}
$$

where the constant factor $[B(\lambda,(1 / q)-\lambda)+B(\lambda,(1 / p)-\lambda)]$ is the best possible.

THEOREM 1.2. If $p>1,1 / p+1 / q=1, f, g \geqslant 0, f \in L^{p}(0, \infty), g \in L^{q}(0, \infty)$ and $\|f\|_{p},\|g\|_{q}>0$, then for $\lambda \geqslant 0$, one has the following two equivalent inequalities:

$$
\begin{gathered}
\int_{0}^{\infty} \int_{0}^{\infty} \frac{(\min \{(x / y),(y / x)\})^{\lambda / 2}}{\max \{x, y\}} f(x) g(y) d x d y<\frac{4 p q(\lambda+1)}{(p \lambda+2)(q \lambda+2)}\|f\|_{p}\left\|_{g}\right\|_{q} \\
\left\{\int_{0}^{\infty}\left(\int_{0}^{\infty} \frac{(\min \{(x / y),(y / x)\})^{\lambda / 2}}{\max \{x, y\}} f(x) d x\right)^{p} d y\right\}^{1 / p}<\frac{4 p q(\lambda+1)}{(p \lambda+2)(q \lambda+2)}\|f\|_{p}
\end{gathered}
$$

where the constant factor $(4 p q(\lambda+1)) /((p \lambda+2)(q \lambda+2))$ is the best possible.

At the same time, Sulaiman (see [9]) gave:

THEOREM 1.3. Let $\ln f(x), \ln g(x)$ be convex for nonnegative functions $f(x)$ and $g(x)$ such that $f(0)=g(0)=0, f(\infty)=g(\infty)=\infty, f^{\prime}(s) \geqslant 0, g^{\prime}(s) \geqslant 0, s \in\left\{x^{p}, y^{q}\right\}$. Let $\lambda>\max \{p, q\}, p>1,1 / p+1 / q=1$. Let

$$
0<\int_{0}^{\infty} \frac{x^{-p^{2} / q^{2}}\left[f\left(x^{p}\right)\right]^{2-\lambda+p / q}}{\left[f^{\prime}\left(x^{p}\right)\right]^{p / q}} d x<\infty, \quad 0<\int_{0}^{\infty} \frac{x^{-q^{2} / p^{2}}\left[g\left(x^{q}\right)\right]^{2-\lambda+q / p}}{\left[g^{\prime}\left(x^{q}\right)\right]^{q / p}} d x<\infty .
$$

Then we have

$$
\begin{aligned}
& \int_{0}^{\infty} \int_{0}^{\infty} \frac{f(x y) g(x y)}{\left(f\left(x^{p}\right), g\left(y^{q}\right)\right)^{\lambda}} d x d y \\
& \leqslant \frac{1}{\sqrt[p]{p} \sqrt[q]{q}} B^{1 / p}(p, \lambda-p) B^{1 / q}(q, \lambda-q)\left\{\int_{0}^{\infty} \frac{x^{-p^{2} / q^{2}}\left[f\left(x^{p}\right)\right]^{2-\lambda+p / q}}{\left[f^{\prime}\left(x^{p}\right)\right]^{p / q}} d x\right\}^{1 / p} \\
&\left.\times \int_{0}^{\infty} \frac{x^{-q^{2} / p^{2}}\left[g\left(x^{q}\right)\right]^{2-\lambda+q / p}}{\left[g^{\prime}\left(x^{q}\right)\right]^{q / p}} d x\right\}^{1 / q}
\end{aligned}
$$

The main purpose of the present article is to establish some new inequalities similar to Hilbert's type inequalities, and the unification of Hardy-Hilbert's and Hardy-Hilbert's type integral inequality. 


\section{MAIN RESULTS AND APPLICATIONS}

THEOREM 2.1. Suppose $f, g$ are nonnegative real functions such that $\int_{1}^{\infty}\left(x^{p}\right.$ $+(1 /(p-1))) f^{p}(x) d x<\infty$ and $\int_{1}^{\infty}\left(x^{q}+(1 /(q-1))\right) g^{q}(x) d x<\infty$ for $p>1,1 / p+1 / q$ $=1$. Then we have

$$
\begin{aligned}
& \int_{1}^{\infty} \int_{1}^{\infty} \frac{f(x) g(y)}{\min \{x, y\}} d x d y \\
& \quad \leqslant \frac{1}{\sqrt[p]{p} \sqrt[q]{q}}\left\{\int_{1}^{\infty}\left(x^{p}+\frac{1}{p-1}\right) f^{p}(x) d x\right\}^{1 / p}\left\{\int_{1}^{\infty}\left(x^{q}+\frac{1}{q-1}\right) g^{q}(x) d x\right\}^{1 / q}
\end{aligned}
$$

where the constant factor $1 /(\sqrt[p]{p} \sqrt[q]{q})$ is the best possible.

Proof: By Hölder's inequality, we have

$$
\begin{aligned}
\int_{1}^{\infty} & \int_{1}^{\infty} \frac{f(x) g(y)}{\min \{x, y\}} d x d y \\
& =\int_{1}^{\infty} \int_{1}^{\infty} \frac{1}{\min \{x, y\}}\left[f(x)\left(\frac{x}{y}\right)\right]\left[g(y)\left(\frac{y}{x}\right)\right] d x d y \\
& \leqslant\left[\int_{1}^{\infty} \int_{1}^{\infty} \frac{f^{p}(x)}{\min \{x, y\}}\left(\frac{x}{y}\right)^{p} d x d y\right]^{1 / p}\left[\int_{1}^{\infty} \int_{1}^{\infty} \frac{g^{q}(y)}{\min \{x, y\}}\left(\frac{y}{x}\right)^{q} d x d y\right]^{1 / q} .
\end{aligned}
$$

Define the weight function $\varpi(x, p)$ as

$$
\varpi(x, p):=\int_{1}^{\infty} \frac{1}{\min \{x, y\}}\left(\frac{x}{y}\right)^{p} d y, x \in[1, \infty),
$$

then the above inequality yields

$$
\int_{1}^{\infty} \int_{1}^{\infty} \frac{f(x) g(y)}{\min \{x, y\}} d x d y \leqslant\left[\int_{1}^{\infty} \varpi(x, p) f^{p}(x) d x\right]^{1 / p}\left[\int_{1}^{\infty} \varpi(y, q) g^{q}(y) d y\right]^{1 / q} .
$$

For fixed $x$, let $y=x t$, we have

$$
\begin{aligned}
\varpi(x, p) & =\int_{1}^{\infty} \frac{1}{\min \{x, y\}}\left(\frac{x}{y}\right)^{p} d y=\int_{1 / x}^{\infty} \frac{1}{\min \{1, t\}} t^{-p} d t \\
& =\int_{1 / x}^{1} t^{-p-1} d t+\int_{1}^{\infty} t^{-p} d t=\frac{1}{p}\left(x^{p}+\frac{1}{p-1}\right),
\end{aligned}
$$

similarly,

$$
\varpi(y, q)=\int_{1}^{\infty} \frac{1}{\min \{x, y\}}\left(\frac{y}{x}\right)^{q} d x=\frac{1}{q}\left(y^{q}+\frac{1}{q-1}\right) .
$$

This shows the right hand side of equality (2.1).

We can prove that there exist nontrivial functions $f(x), g(x)$, such that (2.1) takes the equality. In fact, define

$$
\begin{aligned}
& f(x)=x^{-q}, \text { for } x \in[1, \infty), \\
& g(y)=y^{-p}, \text { for } y \in[1, \infty) .
\end{aligned}
$$


On one hand, we have

$$
\begin{aligned}
& \int_{1}^{\infty} \int_{1}^{\infty} \frac{f(x) g(y)}{\min \{x, y\}} d x d y \\
& \leqslant \frac{1}{\sqrt[p]{p} \cdot q \sqrt{q}}\left\{\int_{1}^{\infty}\left(x^{p}+\frac{1}{p-1}\right) f^{p}(x) d x\right\}^{1 / p}\left\{\int_{1}^{\infty}\left(x^{q}+\frac{1}{q-1}\right) g^{q}(x) d x\right\}^{1 / q} \\
& =\frac{1}{\sqrt[p]{p} \cdot \sqrt[q]{q}}\left\{\int_{1}^{\infty}\left(x^{p}+\frac{1}{p-1}\right) x^{-p q} d x\right\}^{1 / p}\left\{\int_{1}^{\infty}\left(x^{q}+\frac{1}{q-1}\right) x^{-p q} d x\right\}^{1 / q} \\
& =\frac{1}{\sqrt[p]{p} \cdot \sqrt[q]{q}}\left[\int_{1}^{\infty} x^{-q} d x+\frac{1}{p-1} \int_{1}^{\infty} x^{-p q} d x\right]^{1 / p}\left[\int_{1}^{\infty} x^{-p} d x+\frac{1}{q-1} \int_{1}^{\infty} x^{-p q} d x\right]^{1 / q} \\
& =\frac{1}{p(q-1)}+\frac{1}{p(p-1)(p+q-1)} .
\end{aligned}
$$

On the other hand, setting $y=x t$, we find

$$
\begin{aligned}
\int_{1}^{\infty} \int_{1}^{\infty} \frac{f(x) g(y)}{\min \{x, y\}} d x d y & =\int_{1}^{\infty} \int_{1}^{\infty} \frac{x^{-q} y^{-p}}{\min \{x, y\}} d x d y \\
& =\int_{1}^{\infty} x^{-(p+q)} d x \int_{1 / x}^{\infty} \frac{1}{\min \{1, t\}} t^{-p} d t \\
& =\int_{1}^{\infty} x^{-(p+q)}\left[\int_{1 / x}^{1} t^{-p-1} d t+\int_{1}^{\infty} t^{-p} d t\right] d x \\
& =\frac{1}{p} \int_{1}^{\infty} x^{-q} d x+\left(\frac{1}{p-1}-\frac{1}{p}\right) \int_{1}^{\infty} x^{-(p+q)} d x \\
& =\frac{1}{p(q-1)}+\frac{1}{p(p-1)(p+q-1)} .
\end{aligned}
$$

Hence the equality of (2.1) can be attained. This completes the theorem.

Specially, for $p=q=2$, we have:

Corollary 2.2. Suppose $f, g$ are real functions such that $\int_{1}^{\infty}\left(1+x^{2}\right) f^{2}(x) d x$ $<\infty$ and $\int_{1}^{\infty}\left(1+x^{2}\right) g^{2}(x) d x<\infty$. Then we have

$$
\begin{aligned}
\int_{1}^{\infty} \int_{1}^{\infty} \frac{f(x) g(y)}{\min \{x, y\}} d x d y \\
\leqslant \frac{1}{2}\left\{\int_{1}^{\infty}\left(1+x^{2}\right) f^{2}(x) d x\right\}^{1 / 2}\left\{\int_{1}^{\infty}\left(1+x^{2}\right) g^{2}(x) d x\right\}^{1 / 2}
\end{aligned}
$$

where the constant factor $1 / 2$ is the best possible.

THEOREM 2.3. Suppose $f, g$ are real functions such that $\int_{1}^{\infty}\left(1+x^{2 \lambda}\right) x^{1-\lambda} f^{2}(x) d x$ 
$<\infty$ and $\int_{1}^{\infty}\left(1+x^{2 \lambda}\right) x^{1-\lambda} g^{2}(x) d x<\infty$ for $\lambda>0$. Then we have

$$
\begin{aligned}
& \int_{1}^{\infty} \int_{1}^{\infty} \frac{f(x) g(y)}{\min \left\{x^{\lambda}, y^{\lambda}\right\}} d x d y \\
& \quad \leqslant \frac{1}{2 \lambda}\left\{\int_{1}^{\infty}\left(1+x^{2 \lambda}\right) x^{1-\lambda} f^{2}(x) d x\right\}^{1 / 2}\left\{\int_{1}^{\infty}\left(1+x^{2 \lambda}\right) x^{1-\lambda} g^{2}(x) d x\right\}^{1 / 2},
\end{aligned}
$$

where the constant factor $1 / 2 \lambda$ is the best possible.

Proof: The proof is similar to Theorem 2.1, thus we omit the details.

Correspondingly, we have the following theorem for series:

THEOREM 2.4. Suppose $p>1,1 / p+1 / q=1, a_{n} \geqslant 0, b_{n} \geqslant 0(n \geqslant 2)$ such that $0<\sum_{n=2}^{\infty}\left(n^{p}+(1 /(p-1))\right) a_{n}^{p}<\infty$ and $0<\sum_{n=2}^{\infty}\left(n^{q}+(1 /(q-1))\right) b_{n}^{q}<\infty$. Then we have

$$
\sum_{n=2}^{\infty} \sum_{n=2}^{\infty} \frac{a_{m} b_{n}}{\min \{m, n\}}<\frac{1}{\sqrt[p]{p} \sqrt[q]{q}}\left\{\sum_{n=2}^{\infty}\left(n^{p}+\frac{1}{p-1}\right) a_{n}^{p}\right\}^{1 / p}\left\{\sum_{n=2}^{\infty}\left(n^{q}+\frac{1}{q-1}\right) b_{n}^{q}\right\}^{1 / q} .
$$

Proof: By Theorem 2.1, setting

$$
\begin{aligned}
& f(x)=a_{m}, \quad(m-1 \leqslant x<m), \\
& g(y)=a_{n}, \quad(n-1 \leqslant y<n) .
\end{aligned}
$$

Knowing that $1 /(\min \{x, y\})$ is a decreasing function of $x$ and $y$, we observe that

$$
\frac{a_{m} b_{n}}{\min \{m, n\}} \leqslant \int_{m-1}^{m} \int_{n-1}^{n} \frac{f(x) g(y)}{\min \{x, y\}} d x d y
$$

unless $a_{m}=0$ or $b_{n}=0$. Hence

$$
\begin{aligned}
\sum_{n=2}^{\infty} \sum_{n=2}^{\infty} \frac{a_{m} b_{n}}{\min }\{m, n\} \\
\leqslant \int_{1}^{\infty} \int_{1}^{\infty} \frac{f(x) g(y)}{\min \{x, y\}} d x d y \\
\leqslant \frac{1}{\sqrt[p]{p} \sqrt[q]{q}}\left\{\int_{1}^{\infty}\left(x^{p}+\frac{1}{p-1}\right) f^{p}(x) d x\right\}^{1 / p}\left\{\int_{1}^{\infty}\left(x^{q}+\frac{1}{q-1}\right) g^{q}(x) d x\right\}^{1 / q} \\
=\frac{1}{\sqrt[p]{p} \cdot \sqrt[q]{q}}\left\{\sum_{n=2}^{\infty} \int_{n-1}^{n}\left(x^{p}+\frac{1}{p-1}\right) a_{n}^{p} d x\right\}^{1 / p}\left\{\sum_{n=2}^{\infty} \int_{n-1}^{n}\left(x^{q}+\frac{1}{q-1}\right) b_{n}^{q} d x\right\}^{1 / q} \\
<\frac{1}{\sqrt[p]{p} \sqrt[q]{q}}\left\{\sum_{n=2}^{\infty}\left(n^{p}+\frac{1}{p-1}\right) a_{n}^{p}\right\}^{1 / p}\left\{\sum_{n=2}^{\infty}\left(n^{q}+\frac{1}{q-1}\right) b_{n}^{q}\right\}^{1 / q} .
\end{aligned}
$$

This completes the proof. 
TheOREM 2.5. Let $p>1,1 / p+1 / q=1$, let $\ln f(x), \ln g(x)$ be convex for nonnegative real functions $f(x), g(x)$ such that $f(1)=g(1)=1, f(\infty)=g(\infty)=\infty$, $f^{\prime}(t) \geqslant 0, g^{\prime}(t) \geqslant 0, t \in[1, \infty)$ and

$$
\begin{aligned}
& 0<\int_{1}^{\infty} x^{-(p-1)^{2}} f\left(x^{p}\right)\left[f^{\prime}\left(x^{p}\right)\right]^{1-p} g\left(x^{p}\right)\left[f^{p}\left(x^{p}\right)+\frac{1}{p-1}\right] d x<\infty, \\
& 0<\int_{1}^{\infty} x^{-(q-1)^{2}} g\left(x^{q}\right)\left[g^{\prime}\left(x^{q}\right)\right]^{1-q} f\left(x^{q}\right)\left[g^{q}\left(x^{q}\right)+\frac{1}{q-1}\right] d x<\infty
\end{aligned}
$$

Then we have

$$
\begin{aligned}
& \int_{1}^{\infty} \int_{1}^{\infty} \frac{f(x y) g(x y)}{\min \left\{f\left(x^{p}\right), g\left(y^{q}\right)\right\}} d x d y \\
& \leqslant \frac{1}{p q}\left\{\int_{1}^{\infty} x^{-(p-1)^{2}} f\left(x^{p}\right)\left[f^{\prime}\left(x^{p}\right)\right]^{1-p} g\left(x^{p}\right)\left[f^{p}\left(x^{p}\right)+\frac{1}{p-1}\right] d x\right\}^{1 / p} \\
& \quad \times\left\{\int_{1}^{\infty} x^{-(q-1)^{2}} g\left(x^{q}\right)\left[g^{\prime}\left(x^{q}\right)\right]^{1-q} f\left(x^{q}\right)\left[g^{q}\left(x^{q}\right)+\frac{1}{q-1}\right] d x\right\}^{1 / q}
\end{aligned}
$$

In particular, when $p=q=2$, the above inequality reduces to

$$
\begin{aligned}
& \int_{1}^{\infty} \int_{1}^{\infty} \frac{f(x y) g(x y)}{\min \left\{f\left(x^{2}\right), g\left(y^{2}\right)\right\}} d x d y \\
& \leqslant \frac{1}{4}\left\{\int_{1}^{\infty} x^{-1} f\left(x^{2}\right)\left[f^{\prime}\left(x^{2}\right)\right]^{-1} g\left(x^{2}\right)\left[f^{2}\left(x^{2}\right)+1\right] d x\right\}^{1 / 2} \\
& \quad \times\left\{\int_{1}^{\infty} x^{-1} g\left(x^{2}\right)\left[g^{\prime}\left(x^{2}\right)\right]^{-1} f\left(x^{2}\right)\left[g^{2}\left(x^{2}\right)+1\right] d x\right\}^{1 / 2} .
\end{aligned}
$$

Proof: Since $\ln f(x)$ is convex and by Young's inequality: $x y \leqslant x^{p} / p+x^{q} / q$, we have

$$
f(x y)=e^{\ln f(x y)} \leqslant e^{\ln f\left(x^{p} / p+y^{q} / q\right)} \leqslant e^{\left(\ln f\left(x^{p}\right) / p\right)+\left(\ln f\left(y^{q}\right) / q\right)}=f^{1 / p}\left(x^{p}\right) f^{1 / q}\left(y^{q}\right) .
$$

Hence by Hölder's inequality, we get

$$
\begin{aligned}
\int_{1}^{\infty} & \int_{1}^{\infty} \frac{f(x y) g(x y)}{\min \left\{f\left(x^{p}\right), g\left(y^{q}\right)\right\}} d x d y \\
\leqslant & \int_{1}^{\infty} \int_{1}^{\infty} \frac{1}{\min \left\{f\left(x^{p}\right), g\left(y^{q}\right)\right\}}\left[\frac{f^{1+(1 / p)}\left(x^{p}\right) g^{(1 / p)}\left(x^{p}\right)}{g\left(y^{q}\right)} \frac{\left[g^{\prime}\left(y^{q}\right)\right]^{1 / p}}{\left[f^{\prime}\left(x^{p}\right)\right]^{1 / q}}\left(\frac{y^{(q-1 / p)}}{x^{(p-1) / q}}\right)\right] \\
& \times\left[\frac{g^{1+(1 / q)}\left(y^{q}\right) f^{1 / q}\left(y^{q}\right)}{f\left(x^{p}\right)} \frac{\left[f^{\prime}\left(x^{p}\right)\right]^{1 / q}}{\left.\left[g^{\prime}\left(y^{q}\right)\right]^{1 / p}\left(\frac{x^{(p-1) / q}}{y^{(q-1) / p}}\right)\right] d x d y}\right. \\
\leqslant & \int_{1}^{\infty} \int_{1}^{\infty} \frac{1}{\min \left\{f\left(x^{p}\right), g\left(y^{q}\right)\right\}}\left[\frac{f^{p+1}\left(x^{p}\right) g\left(x^{p}\right)}{g^{p}\left(y^{q}\right)} \frac{g^{\prime}\left(y^{q}\right)}{\left[f^{\prime}\left(x^{p}\right)\right]^{p / q}}\left(\frac{y^{q-1}}{x^{(p(p-1)) / q}}\right)\right] d x d y \\
& \times \int_{1}^{\infty} \int_{1}^{\infty} \frac{1}{\min \left\{f\left(x^{p}\right), g\left(y^{q}\right)\right\}}\left[\frac{g^{q+1}\left(y^{q}\right) f\left(y^{q}\right)}{f^{q}\left(x^{p}\right)} \frac{\left[f^{\prime}\left(x^{p}\right)\right]}{\left[g^{\prime}\left(y^{q}\right)\right]^{q / p}}\left(\frac{x^{p-1}}{y^{(q(q-1)) / p}}\right)\right] d x d y
\end{aligned}
$$




$$
\begin{aligned}
& =\left\{\int_{1}^{\infty} \frac{1}{q} x^{-(p-1)^{2}} f\left(x^{p}\right)\left[f^{\prime}\left(x^{p}\right)\right]^{1-p} g\left(x^{p}\right)\left[\int_{1}^{\infty} \frac{q y^{q-1} g^{\prime}\left(y^{q}\right)}{\min \left\{f\left(x^{p}\right), g\left(y^{q}\right)\right\}}\left(\frac{f\left(x^{p}\right)}{g\left(y^{q}\right)}\right)^{p} d y\right] d x\right\}^{1 / p} \\
& (2.7) \times\left\{\int_{1}^{\infty} \frac{1}{p} y^{-(q-1)^{2}} g\left(y^{q}\right)\left[g^{\prime}\left(y^{q}\right)\right]^{1-q} f\left(y^{q}\right)\left[\int_{1}^{\infty} \frac{p x^{p-1} f^{\prime}\left(x^{p}\right)}{\min \left\{f\left(x^{p}\right), g\left(y^{q}\right)\right\}}\left(\frac{g\left(y^{q}\right)}{f\left(x^{p}\right)}\right)^{q} d x\right] d y\right\}^{1 / q} .
\end{aligned}
$$

Define the weight function $\varphi(x, p), \psi(y, q)$ as

$$
\begin{aligned}
& \varphi(x, p):=\int_{1}^{\infty} \frac{q y^{q-1} g^{\prime}\left(y^{q}\right)}{\min \left\{f\left(x^{p}\right), g\left(y^{q}\right)\right\}}\left(\frac{f\left(x^{p}\right)}{g\left(y^{q}\right)}\right)^{p} d y, x \in[1, \infty) \\
& \psi(y, q):=\int_{1}^{\infty} \frac{p x^{p-1} f^{\prime}\left(x^{p}\right)}{\min \left\{f\left(x^{p}\right), g\left(y^{q}\right)\right\}}\left(\frac{g\left(y^{q}\right)}{f\left(x^{p}\right)}\right)^{q} d x
\end{aligned}
$$

then the above inequality yields

$$
\begin{aligned}
& \int_{1}^{\infty} \int_{1}^{\infty} \frac{f(x y) g(x y)}{\min \left\{f\left(x^{p}\right), g\left(y^{q}\right)\right\}} d x d y \\
& \leqslant\left[\int_{1}^{\infty} \frac{1}{q} \varphi(x, p) x^{-(p-1)^{2}} f\left(x^{p}\right)\left[f^{\prime}\left(x^{p}\right)\right]^{1-p} g\left(x^{p}\right) d x\right]^{1 / p} \\
& \times\left[\int_{1}^{\infty} \frac{1}{p} \psi(y, q) y^{-(q-1)^{2}} g\left(y^{q}\right)\left[g^{\prime}\left(y^{q}\right)\right]^{1-q} f\left(y^{q}\right) d y\right]^{1 / q}
\end{aligned}
$$

Similar to Theorem 2.1, we have

$$
\begin{aligned}
& \varphi(x, p)=\int_{1}^{\infty} \frac{q y^{q-1} g^{\prime}\left(y^{q}\right)}{\min \left\{f\left(x^{p}\right), g\left(y^{q}\right)\right\}}\left(\frac{f\left(x^{p}\right)}{g\left(y^{q}\right)}\right)^{p} d y=\frac{1}{p}\left[f^{p}\left(x^{p}\right)+\frac{1}{p-1}\right], \\
& \psi(y, q)=\int_{1}^{\infty} \frac{p x^{p-1} f^{\prime}\left(x^{p}\right)}{\min \left\{f\left(x^{p}\right), g\left(y^{q}\right)\right\}}\left(\frac{g\left(y^{q}\right)}{f\left(x^{p}\right)}\right)^{q} d x=\frac{1}{q}\left[g^{q}\left(y^{q}\right)+\frac{1}{q-1}\right] .
\end{aligned}
$$

Hence we obtain equality (2.6). This completes the theorem.

THEOREM 2.6. Suppose $p>1,1 / p+1 / q=1$. Let $\ln f(x), \ln g(x)$ be convex for nonnegative real functions $f(x), g(x)$ such that $\int_{1}^{\infty}\left(x^{p}+(1 /(p-1))\right) f\left(x^{p}\right) d x<\infty$ and $\int_{1}^{\infty}\left(x^{q}+(1 /(q-1))\right) g\left(x^{q}\right) d x<\infty$. Then we have

$$
\begin{aligned}
& \int_{1}^{\infty} \int_{1}^{\infty} \frac{f(x y) g(x y)}{\min \{x, y\}} d x d y \\
\leqslant & \frac{1}{\sqrt[p]{p} \cdot \sqrt[q]{q}}\left\{\int_{1}^{\infty}\left(x^{p}+\frac{1}{p-1}\right) f\left(x^{p}\right) g\left(x^{p}\right) d x\right\}^{1 / p}\left\{\int_{1}^{\infty}\left(x^{q}+\frac{1}{q-1}\right) f\left(x^{q}\right) g\left(x^{q}\right) d x\right\}^{1 / q} .
\end{aligned}
$$

Proof: Since $\ln f(x)$ is convex and $x y \leqslant\left(x^{p}\right) / p+\left(x^{q}\right) / q$, then

$$
f(x y)=e^{\ln f(x y)} \leqslant e^{\ln f\left(x^{p} / p\right)+\left(y^{p} / q\right)} \leqslant e^{\left(\ln f\left(x^{p}\right) / p\right)+\left(\ln f\left(y^{q}\right) / q\right)}=f^{1 / p}\left(x^{p}\right) f^{1 / q}\left(y^{q}\right) .
$$


Therefore, applying Hölder's inequality, we have

$$
\begin{aligned}
& \int_{1}^{\infty} \int_{1}^{\infty} \frac{f(x y) g(x y)}{\min \{x, y\}} d x d y \\
& \quad \leqslant \int_{1}^{\infty} \int_{1}^{\infty} \frac{1}{\min \{x, y\}}\left[f^{1 / p}\left(x^{p}\right) g^{1 / p}\left(x^{p}\right)\left(\frac{x}{y}\right)\right]\left[f^{1 / q}\left(y^{q}\right) g^{1 / q}\left(y^{q}\right)\left(\frac{y}{x}\right)\right] d x d y \\
& \quad \leqslant\left[\int_{1}^{\infty} \int_{1}^{\infty} \frac{f\left(x^{p}\right) g\left(x^{p}\right)}{\min \{x, y\}}\left(\frac{x}{y}\right)^{p} d x d y\right]^{1 / p}\left[\int_{1}^{\infty} \int_{1}^{\infty} \frac{f\left(y^{q}\right) g\left(y^{q}\right)}{\min \{x, y\}}\left(\frac{y}{x}\right)^{q} d x d y\right]^{1 / q} .
\end{aligned}
$$

Define the weight function $\varpi(x, p)$ as

$$
\varpi(x, p):=\int_{1}^{\infty} \frac{1}{\min \{x, y\}}\left(\frac{x}{y}\right)^{p} d y, x \in[1, \infty)
$$

then the above inequality yields

$$
\int_{1}^{\infty} \int_{1}^{\infty} \frac{f(x) g(y)}{\min \{x, y\}} d x d y \leqslant\left[\int_{1}^{\infty} \varpi(x, p) f\left(x^{p}\right) g\left(x^{p}\right) d x\right]^{1 / p}\left[\int_{1}^{\infty} \varpi(y, q) f\left(y^{q}\right) g\left(y^{q}\right) d y\right]^{1 / q} .
$$

The rest of the proof can be completed by following the same steps as in the proof of Theorem 2.1, we get (2.8).

Now we turn to introduce the unification of Hardy-Hilbert's and Hardy-Hilbert's type integral inequality. Some lemmas are given first:

Lemma 2.7. Suppose $r>1,1 / r+1 / s=1, A>0, A+B \geqslant 0$, define the weight function $\varpi(x, s)$ as

$$
A(x+y)+B \min \{x, y\}\left(\frac{x}{y}\right)^{1 / s} d y, \quad x \in(0, \infty),
$$

setting $\varpi(x, s)=C(A, B, s)$, where $C(A, B, s)$ is a constant. Then

$$
0<C(A, B, s)<\infty \text {. }
$$

In particular,

$$
C(1,0, r)=\frac{\pi}{\sin (\pi / r)}, \quad C(1,-1, r)=\frac{r^{2}}{r-1} .
$$

Proof: For fixed $x$, letting $t=y / x$ and $A>0, A+B>0$, we get

$$
\begin{aligned}
\varpi(x, s) & =\int_{0}^{\infty} \frac{1}{A(x+y)+B \min \{x, y\}}\left(\frac{x}{y}\right)^{1 / s} d y \\
& =\int_{0}^{\infty} \frac{1}{A(1+t)+B \min \{1, t\}} t^{-1 / s} d t \\
& =\int_{0}^{1} \frac{1}{A(1+t)+B t} t^{-1 / s} d t+\int_{1}^{\infty} \frac{1}{A(1+t)+B} t^{-1 / s} d t
\end{aligned}
$$




$$
\begin{aligned}
& =\frac{1}{A^{1 / s}(A+B)^{1 / r}} \int_{0}^{(A+B) / A} \frac{1}{1+t} t^{-1 / s} d t+\frac{1}{A^{1 / r}(A+B)^{1 / s}} \int_{A /(A+B)}^{\infty} \frac{1}{1+t} t^{-1 / s} d t \\
& \leqslant \frac{1}{A^{1 / s}(A+B)^{1 / r}} \int_{0}^{\infty} \frac{1}{1+t} t^{-1 / s} d t+\frac{1}{A^{1 / r}(A+B)^{1 / s}} \int_{0}^{\infty} \frac{1}{1+t} t^{-1 / s} d t \\
& =\left[\frac{1}{A^{1 / s}(A+B)^{1 / r}}+\frac{1}{A^{1 / r}(A+B)^{1 / s}}\right] B\left(\frac{1}{r}, \frac{1}{s}\right)<\infty .
\end{aligned}
$$

Hence $0<C(A, B, s)<\infty$.

In particular, we have the following results directly:

$$
\begin{aligned}
C(1,0, r) & =\int_{0}^{\infty} \frac{1}{x+y}\left(\frac{y}{x}\right)^{1 / r} d x=\int_{0}^{\infty} \frac{1}{1+t} t^{-(1 / r)} d t=B\left(\frac{1}{r}, \frac{1}{s}\right)=\frac{\pi}{\sin (\pi / r)} \\
C(1,-1, r) & =\int_{0}^{\infty} \frac{1}{\max \{x, y\}}\left(\frac{y}{x}\right)^{1 / r} d x=\int_{0}^{\infty} \frac{1}{\max \{1, t\}} t^{-\frac{1}{r}} d t=\frac{r^{2}}{r-1}
\end{aligned}
$$

Lemma 2.8. Suppose $r>1,1 / r+1 / s=1$ and $A>0, A+B \geqslant 0, \varepsilon>0$. Then we have

$$
\int_{1}^{\infty} x^{-\varepsilon-1} \int_{0}^{1 / x} \frac{1}{A(1+t)+B \min \{1, t\}} t^{(-1-\varepsilon) / s} d t d x=O(1)\left(\varepsilon \rightarrow 0^{+}\right)
$$

Proof: For $\varepsilon \in(0,(s /(2 r))$ and $x \geqslant 1$, we have

$$
\begin{aligned}
& \int_{0}^{1 / x} \frac{1}{A(1+t)+B \min \{1, t\}} t^{(-1-\varepsilon) / s} d t \\
& \leqslant \frac{1}{A} \int_{0}^{1 / x} t^{(-1-\varepsilon) / s} d t=\frac{1}{A(1+(-1-\varepsilon) / s)}\left(\frac{1}{x}\right)^{1+(-1-\varepsilon) / s} .
\end{aligned}
$$

Since for $a \geqslant 1$ the function $g(y)=\left(1 /\left(y a^{y}\right)\right) \quad(y \in(0, \infty))$ is decreasing, we find

$$
\frac{1}{1+(-1-\varepsilon / s)}\left(\frac{1}{x}\right)^{1+(-1-\varepsilon) / s} \leqslant \frac{1}{1+(-1-s /(2 r)) / s}\left(\frac{1}{x}\right)^{1+(-1-s /(2 r)) / s} \leqslant 2 r\left(\frac{1}{x}\right)^{1 /(2 r)}
$$

so

$$
\begin{aligned}
0 & <\int_{1}^{\infty} x^{-\varepsilon-1} \int_{0}^{1 / x} \frac{1}{A(1+t)+B \min \{1, t\}} t^{(-1-\varepsilon) / s} d t d x \\
& \leqslant \frac{2 r}{A} \int_{1}^{\infty} x^{-1}\left(\frac{1}{x}\right)^{1 /(2 r)} d x \\
& =\frac{4 r^{2}}{A} .
\end{aligned}
$$

Hence relation (2.11) is valid. The lemma is proved. 
ThEOREM 2.9. Suppose $f(x), g(x) \geqslant 0, p>1,1 / p+1 / q=1, A>0, A+B \geqslant 0$, $0<\int_{0}^{\infty} f^{p}(x) d x<\infty$ and $0<\int_{0}^{\infty} g^{q}(x) d x<\infty$. Then

$$
\begin{aligned}
& \int_{0}^{\infty} \int_{0}^{\infty} \frac{f(x) g(y)}{A(x+y)+B \min \{x, y\}} d x d y \\
&<C(A, B, p)\left\{\int_{0}^{\infty} f^{p}(x) d x\right\}^{1 / p}\left\{\int_{0}^{\infty} g^{q}(x) d x\right\}^{1 / q},
\end{aligned}
$$

where the constant factor $C(A, B, p)$ is the best possible. In particular,

(i) for $A=1, B=0$, it reduces to:

$$
\int_{0}^{\infty} \int_{0}^{\infty} \frac{f(x) g(y)}{x+y} d x d y<\frac{\pi}{\sin (\pi / p)}\left\{\int_{0}^{\infty} f^{p}(x) d x\right\}^{1 / p}\left\{\int_{0}^{\infty} g^{q}(x) d x\right\}^{1 / q}
$$

(ii) for $A=1, B=-1$, it reduces to:

$$
\int_{0}^{\infty} \int_{0}^{\infty} \frac{f(x) g(y)}{\max \{x, y\}} d x d y<p q\left\{\int_{0}^{\infty} f^{p}(x) d x\right\}^{1 / p}\left\{\int_{0}^{\infty} g^{q}(x) d x\right\}^{1 / q} .
$$

Proof: (1) For $B=0$ or $A+B=0$, we have (2.12a) and $(2,12 \mathrm{~b})$ respectively.

(2) For $A>0, A+B>0$, by Hölder's inequality and Lemma 2.7, we obtain

$$
\begin{aligned}
\int_{0}^{\infty} & \int_{0}^{\infty} \frac{f(x) g(y)}{A(x+y)+B \min \{x, y\}} d x d y \\
& =\int_{0}^{\infty} \int_{0}^{\infty} \frac{1}{A(x+y)+B \min \{x, y\}}\left[f(x)\left(\frac{x}{y}\right)^{1 / p q}\right]\left[g(y)\left(\frac{y}{x}\right)^{1 / p q}\right] d x d y \\
& \leqslant\left\{\int_{0}^{\infty} \int_{0}^{\infty} \frac{f^{p}(x)}{A(x+y)+B \min \{x, y\}}\left(\frac{x}{y}\right)^{1 / q} d x d y\right\}^{1 / p} \\
& \times\left\{\int_{0}^{\infty} \int_{0}^{\infty} \frac{g^{q}(y)}{A(x+y)+B \min \{x, y\}}\left(\frac{y}{x}\right)^{1 / p} d x d y\right\}^{1 / q} . \\
= & =\left\{(A, B, p)\left\{\int_{0}^{\infty} \varpi(x, q) f^{p}(x) d x\right\}^{1 / p}\left\{\int_{0}^{\infty} \varpi(y, p) g^{q}(y) d y\right\}^{1 / q}\right.
\end{aligned}
$$

This shows the right hand side of (2.12).

If (2.13) takes the form of the equality, then there exist constants a and b, such that they are not all zero and (see [5])

$$
a f^{p}(x)\left(\frac{x}{y}\right)^{1 / q}=b g^{q}(y)\left(\frac{y}{x}\right)^{1 / p}
$$

Then we have

$$
a x f^{p}(x)=b y g^{q}(y), \quad \text { almost everywhere on }(0, \infty) \times(0, \infty),
$$


Hence there exist a constant $d$, such that

$$
a x f^{p}(x)=b y g^{q}(y)=d, \quad \text { almost everywhere on }(0, \infty) \times(0, \infty) .
$$

Without losing the generality, suppose $a \neq 0$, then we obtain $f^{p}(x)=d /(a x)$, almost everywhere on $(0, \infty)$, which contradicts the fact that

$$
0<\int_{0}^{\infty} f^{p}(x) d x<\infty
$$

Hence (2.13) takes the form of strict inequality, we get (2.12).

For $\varepsilon>0$ sufficiently small, setting $f_{\varepsilon}(x)=x^{(-\varepsilon-1) / p}$, for $x \in[1, \infty) ; f_{\varepsilon}(x)=0$, for $x \in(0,1)$ and $g_{\varepsilon}(y)=y^{(-\varepsilon-1) / q}$, for $y \in[1, \infty) ; g_{\varepsilon}(y)=0$, for $y \in(0,1)$. Assume that the constant factor $C(A, B, p)$ in (2.12) is not the best possible, then there exist a positive real number $K$ with $K<C(A, B, p)$, such that (2.12) is valid by changing $C(A, B, p)$ to $K$. On one hand, we have

$$
\int_{0}^{\infty} \int_{0}^{\infty} \frac{f(x) g(y)}{A(x+y)+B \min \{x, y\}} d x d y<K\left\{\int_{0}^{\infty} f_{\varepsilon}^{p}(x) d x\right\}^{1 / p}\left\{\int_{0}^{\infty} g_{\varepsilon}^{q}(y) d y\right\}^{1 / q}=K / \varepsilon
$$

On the other hand, setting $t=y / x$, by Lemma 2.8 , we have

$$
\begin{aligned}
\int_{0}^{\infty} \int_{0}^{\infty} \frac{f(x) g(y)}{A(x+y)+B \min \{x, y\}} d x d y \\
\quad=\int_{1}^{\infty} \int_{1}^{\infty} \frac{x^{(-\varepsilon-1) / p} y^{(-\varepsilon-1) / q}}{A(x+y)+B \min \{x, y\}} d x d y \\
=\int_{1}^{\infty} x^{-\varepsilon-1} \int_{1 / x}^{\infty} \frac{1}{A(1+t)+B \min \{1, t\}} t^{(-1-\varepsilon) / q} d t d x \\
=\int_{1}^{\infty} x^{-\varepsilon-1} \int_{0}^{\infty} \frac{1}{A(1+t)+B \min \{1, t\}} t^{(-1-\varepsilon) / q} d t d x \\
\quad \quad-\int_{1}^{\infty} x^{-\varepsilon-1} \int_{0}^{1 / x} \frac{1}{A(1+t)+B \min \{1, t\}} t^{(-1-\varepsilon) / q} d t d x \\
=\frac{1}{\varepsilon}[C(A, B, p)+\circ(1)]-O(1) \\
=\frac{1}{\varepsilon}[C(A, B, p)+o(1)] .
\end{aligned}
$$

Then we get $(1 / \varepsilon)[C(A, B, p)+o(1)] \leqslant K / \varepsilon$, that is, $C(A, B, p) \leqslant K$ when $\varepsilon$ is sufficiently small, which contradicts the hypothesis. Hence the constant factor $C(A, B, p)$ in (2.12) is the best possible.

THEOREM 2.10. Suppose $f \geqslant 0, p>1,1 / p+1 / q=1, A>0, A+B \geqslant 0$ and $0<\int_{0}^{\infty} f^{p}(x) d x<\infty$. Then

$$
\int_{0}^{\infty}\left[\int_{0}^{\infty} \frac{f(x)}{A(x+y)+B \min \{x, y\}} d x\right]^{p} d y<C^{p}(A, B, p) \int_{0}^{\infty} f^{p}(x) d x
$$


where the constant factor $C^{p}(A, B, p)$ is the best possible. Inequality (2.14) is equivalent to $(2.12)$.

PROOF: Setting $g(y)$ as

$$
\left[\int_{0}^{\infty} \frac{f(x)}{A(x+y)+B \min \{x, y\}} d x\right]^{p-1}, \quad y \in(0, \infty),
$$

then by $(2.12)$, we find

$$
\begin{aligned}
0<\int_{0}^{\infty} g^{q}(y) d y & =\int_{0}^{\infty}\left[\int_{0}^{\infty} \frac{f(x)}{A(x+y)+B \min \{x, y\}} d x\right]^{p} d y \\
& =\int_{0}^{\infty} \int_{0}^{\infty} \frac{f(x) g(y)}{A(x+y)+B \min \{x, y\}} d x d y \\
& \leqslant C(A, B, p)\left\{\int_{0}^{\infty} f^{p}(x) d x\right\}^{1 / p}\left\{\int_{0}^{\infty} g^{q}(y) d y\right\}^{1 / q} .
\end{aligned}
$$

Hence we obtain

$$
0<\int_{0}^{\infty} g^{q}(y) d y \leqslant C^{p}(A, B, p) \int_{0}^{\infty} f^{p}(x) d x<\infty .
$$

By (2.12), both (2.15) and (2.16) take the form of strict inequality, so we have (2.14). On the other hand, suppose that (2.14) is valid. By Hölder's inequality, we find

$$
\begin{gathered}
\int_{0}^{\infty} \int_{0}^{\infty} \frac{f(x) g(y)}{A(x+y)+B \min \{x, y\}} d x d y \\
\quad=\int_{0}^{\infty}\left[\int_{0}^{\infty} \frac{f(x)}{A(x+y)+B \min \{x, y\}} d x\right] g(y) d y \\
\quad \leqslant\left\{\int_{0}^{\infty}\left[\int_{0}^{\infty} \frac{f(x)}{A(x+y)+B \min \{x, y\}} d x\right]^{p} d y\right\}^{1 / p}\left\{\int_{0}^{\infty} g^{q}(y) d y\right\}^{1 / q} .
\end{gathered}
$$

Then by (2.14), we have (2.12). Thus (2.12) and (2.14) are equivalent.

If the constant factor $C^{p}(A, B, p)$ in (2.14) is not the best possible, by (2.17), we may get a contradiction that the constant factor in (2.12) is not the best possible. Thus we complete the proof of the theorem.

REMARK 2.1. (i) for $A=1, B=0$, inequality (2.14) reduces to the equivalent form of Hardy-Hilbert's inequality:

$$
\int_{0}^{\infty}\left[\int_{0}^{\infty} \frac{f(x)}{x+y} d x\right]^{p} d y<\left[\frac{\pi}{\sin (\pi / p)}\right]^{p} \int_{0}^{\infty} f^{p}(x) d x
$$

(ii) for $A=1, B=-1$, inequality (2.14) reduces to the equivalent form of Hardy-Hilbert's type inequality:

$$
\int_{0}^{\infty}\left[\int_{0}^{\infty} \frac{f(x)}{\max \{x, y\}} d x\right]^{p} d y<(p q)^{p} \int_{0}^{\infty} f^{p}(x) d x
$$

where both the constant factors $[\pi /(\sin (\pi / p))]^{p}$ and $(p q)^{p}$ are the best possible. 


\section{REFERENCES}

[1] Y.C. Chow, 'On inequalities of Hilbert and Widder', J. London Math. Soc. 14 (1939), 151-154.

[2] M. Gao, 'On Hilbert's inequality and its applications', J. Math. Anal. Appl. 212 (1997), 316-323.

[3] G.H. Hardy, 'Note on a theorem of Hilbert', Math. Z. 6 (1920), 314-317.

[4] G.H. Hardy, J.E. Littlewood and G. Polya, Inequalities (Cambridge University Press, Cambridge, 1934).

[5] J. Kuang, 'Applied inequalities', (in Chinese), Second edition (Hunan Education Press, Changsha).

[6] J. Kuang, 'On new extensions of Hilbert's integral inequality', Math. Anal. Appl. 235 (1999), 608-614.

[7] D.S. Mitrinovic, J.E. Pecaric and A.M. Fink, Inequalities involving functions and their integrals and derivatives (Kluwer Academic, Boston, 1991).

[8] B.G. Pachpatte, 'Inequalities similar to the integral analogue of Hilbert's inequality', Tamkang J. Math. 30 (1999), 139-146.

[9] W.T. Sulaiman, 'Four inequalities similar to Hardy-Hilbert's integral inequality', JIPAM. J. Inequal. Pure Appl. Math. 7 (2006), Article 76, 8 pp.

[10] B. Yang, 'An extension of Hardy-Hilbert's inequality', (in Chinese), Chinese Ann. Math. Ser. A 23 (2002), 247-254.

[11] B. Yang, 'On the norm of an integral operator and applications', J. Math. Anal. Appl. 321 (2006), 182-192.

Department of Mathematics

Sun Yat-Sen University

Guangzhou 510275

People's Republic of China

e-mail: stslyj@mail.sysu.edu.cn
Department of Mathematics

Guangdong Education College

Guangzhou 510303

People's Republic China

e-mail: hzs314@163.com 